\title{
Justicia ambiental y gestión de los bienes comunes
}

\author{
Environmental Justice and Common Goods Management
}

\section{Tom Angotti ${ }^{1}$}

\begin{abstract}
La problemática de la justicia ambiental tiene sus raíces históricas en la colonización de las Américas, tanto en el norte como en el centro y sur. Desde la época de la colonia la injusticia ambiental tuvo sus raíces en la relación de los seres humanos con la tierra y precisamente en la explotación sin límites de la tierra por parte de los colonos europeos. Éstos ocupaban la tierra para facilitar la extracción de los recursos que servían al mercado europeo, las empresas industriales y mineras, las compañías mercantiles y más adelante las instituciones financieras dedicadas a la circulación y reproducción del capital. Esta larga cadena económica empezó con el impulso inicial de la explotación sin escrúpulos de la tierra, el inicio del capitalismo extractivo. Con la famosa frase del Libertador Simón Bolivar: "Si la naturaleza se opone, lucharemos contra ella y haremos que nos obedezca", como expresión de absoluta confianza en la capacidad humana para dominar la tierra, se está frente al argumento político a favor de la explotación minera que habían empezado los regímenes coloniales y que se continuaban después de la independencia a través de la institución del latifundio.
\end{abstract}

En la actualidad, en el mundo entero crece la preocupación por los impactos del cambio climático y en línea con los acuerdos internacionales alcanzados en Río de Janeiro en 1992, hasta el último en la Conferencia de París, una nueva generación de expertos, científicos y ciudadanos han comenzado a buscar soluciones racionales que puedan ser implementadas por los gobiernos locales, nacionales y las instituciones internacionales.

\footnotetext{
${ }^{1}$ Hunter College y del Graduate Center, City University of New York. tangotti@ hunter.cuny.edu
} 
Estamos frente a una abundancia de posibles soluciones, tanto tecnológicas como científicas. El problema radica en que, al implementarse las nuevas medidas, se alienta a las minorías más poderosas del mundo, aquellas que han creado los problemas bajo el modelo del capitalismo consumista y salvaje, mientras los que tienen menos recursos siguen siendo las víctimas, sufriendo las consecuencias del cambio climático. Esta situación es lo que denominamos hoy en día un problema de justicia ambiental, a analizar críticamente.

Palabras clave: cambio climático, modelo capitalista, justicia ambiental.

The problem of environmental justice has its historical roots in the colonization of the Americas, both in the north and in the center and south. From the time of the colony environmental injustice had its roots in the relationship of human beings with the earth and precisely in the unlimited exploitation of the land by European settlers. They occupied the land to facilitate the extraction of the resources that served the European market, the industrial and mining companies, the mercantile companies and later the financial institutions dedicated to the circulation and reproduction of the capital. This long economic chain began with the initial impulse of the unscrupulous exploitation of the land, the beginning of extractive capitalism. With the famous phrase of the Liberator Simón Bolívar: "If nature opposes, we will fight against it and make it obey us", as an expression of absolute confidence in the human capacity to dominate the earth, we are facing the political argument in favor of the mining that the colonial regimes had begun and that continued after independence through the institution of the latifundio.

Nowadays, worldwide concern about the impacts of climate change is growing and in line with the international agreements reached in Rio de Janeiro in 1992, until the last one at the Paris Conference, a new generation of experts, scientists and Citizens have begun to seek rational solutions that can be implemented by local and national governments and international institutions.

We are facing an abundance of possible solutions, both technological and scientific. The problem is that, when the new measures are implemented, the most powerful minorities in the world are encouraged, those that have created the problems under the model of savage consumerist capitalism, while those with fewer resources continue to be the victims, suffering the consequences of climate change. This situation is what we call today a problem of environmental justice, to analyze critically.

Keywords: Climate change, capitalist model, environmental justice 


\section{NUESTRA RELACIÓN CON LA TIERRA}

Uno de los grandes mitos en Norteamérica sostiene que la economía y política que implantaban los ingleses y franceses en el norte fue más racional y respetuosa en el uso y cuidado de la tierra con un régimen que garantizaba un desarrollo tal que favorecía a la mayoría de las poblaciones humanas mientras el desarrollo del Sur fue siempre más desigual. La dimensión de la pobreza en el Sur y la hegemonía del pensamiento racional y tecnocrático entre las élites de todas las Américas, crearon la base para estos mitos. Basta con recordar que en los Estados Unidos y en Canadá los colonos europeos ocuparon las tierras que antes habían pertenecido a los indígenas a través de operaciones genocidas. Estas tierras fueron transformadas en grandes extensiones agrícolas y ganaderas que contaminaron la tierra y las aguas con fertilizantes e insecticidas químicos e iniciaron la explotación del petróleo y el gas natural. Hoy en día, en que se informa que los Estados Unidos se encamina a ser el país de mayor producción de petróleo y gas en el mundo, es momento de reflexionar cómo la historia de expansión imperial del norte y su control de los suelos, los subsuelos y el espacio, ha hecho posible el dramático cambio climático que conocemos en todo el mundo.

En la actualidad, resulta fácil olvidar que en América nuestra relación con la tierra ha sido impulsada por intermedio de la violencia de los poderes militares. Estados Unidos empezó con trece estados en el este, siendo ahora un país con cincuenta estados. Al inicio del siglo XX, su extensión llegó mucho más allá del continente con invasiones a Puerto Rico, Las Filipinas y Hawai. Cuenta con la fuerza militar más grande del mundo, presente en más de cien países y un presupuesto militar que se encuentra a la cabeza de los seis presupuestos mayores del mundo y un complejo militar que es el más contaminante.

La violencia militar y de la policía nacional en América Latina ha sido un elemento fundamental en el proceso de desplazamiento de la población de las áreas rurales hacia las grandes metrópolis. Este rápido y muchas veces violento proceso de urbanización, no responde simplemente a un proceso "natural" de migración campo-ciudad. El proceso ha sido conscientemente organizado por las fuerzas económicas, políticas y militares. El desplazamiento no ha terminado con la formación de las grandes metrópolis, sino que ahora se multiplica por el desalojo de los barrios pobres y de la clase media, impulsado por el ingreso y especulaciones de los capitales financieros a nivel global (Angotti, 2013; 2017).

\section{EL TERRITORIO METROPOLITANO}

El modelo de explotación del territorio rural se adaptó a los centros urbanos. En el siglo XXI, las Américas están casi enteramente urbanizadas, en un mundo donde por primera vez la mayoría de la población global vive en grandes ciudades, las metrópolis.

La metrópoli americana nació bajo la misma política de desarrollo del territorio que antes se aplicaba a los medios rurales. La tierra urbana fue objeto de un capitalismo extractivo, 
donde se usa la tierra urbana como mercancía a favor de los propietarios y para el fomento del negocio de bienes raíces. Los urbanistas y planificadores modernos son los expertos en "el uso del suelo," un concepto totalmente ajeno a las culturas indigenas y pre-colombinas donde la tierra era sagrada, una entidad viva e integrada con la vida natural. En los núcleos centrales de las metrópolis, donde el valor del suelo es mayor, se generan presiones que desencadenan el desplazamiento de las poblaciones con menores recursos económicos. Muchas veces los mismos refugiados de las áreas rurales, son los que pagan con el desalojo urbano como antes lo habían hecho con el desalojo rural.

Otra característica de la metrópoli es su división en enclaves aislados, donde las divisiones de clase, de etnia y de raza determinan el mercado de tierras y sus precios. Los estados americanos no han asumido la responsabilidad de invertir en el mejoramiento de los barrios "informales" pero cuando sube el precio del suelo en los principales barrios, el estado procede a su eliminación y al "mejoramiento" por medio de la construcción de nuevas urbanizaciones de infraestructura moderna para las clases medias y altas.

La metrópoli moderna es la del consumo, con pocos límites impuestos a la producción y circulación de los productos comerciales. A medida que se va generando el exceso de capital, la metrópoli se colma de artículos de consumo, pero también de desechos contaminantes. Un hecho singular del siglo XX fue el negocio de los "desechos" y del "efluente." En suma, el suelo urbano es un producto que se consume, al servicio de los seres humanos y que puede desecharse como cualquier otro producto, cuando ya no es útil (Angotti, 2012).

\section{EL CAMBIO CLIMÁTICO Y LA JUSTICIA AMBIENTAL}

El territorio metropolitano ha sido siempre objeto de la máxima explotación por parte de los seres humanos. A finales del siglo XXI, si no se producen fuertes cambios económicos, políticos y económicos, la casi totalidad de la población humana va a vivir en las metrópolis, en tanto las áreas periurbanas estarán sujetas a una explotación más intensa de recursos minerales y una agricultura más industrializada y contaminante. La destrucción del territorio periurbano puede ser aún más catastrófica por su invisibilidad con respecto a la mayoría de la humanidad.

La metrópoli como entidad urbana, comparada con los asentamientos urbanos de pequeña extensión, emite más de $80 \%$ del carbono a la atmósfera del mundo. El vehículo privado es el principal medio de transporte en las metrópolis (responsable del $25 \%$ del carbono emitido), la energía utilizada es responsable de alrededor del 50\% del carbono y ellas son también las principales consumidoras de los productos derivados de la agricultura industrial, responsables del $25 \%$ de los gases de efecto invernadero a nivel mundial. Además, el nivel de consumo per cápita de los productos industriales en las metrópolis y el nivel de desechos contaminantes son varias veces más altos que en las áreas rurales y los asentamientos más pequeños. 
Hay quienes teorizan que la urbanización total del planeta será la salvación. Afirman que el problema más agudo del siglo XXI, el calentamiento global, será más manejable porque se trata de un territorio que en su totalidad no llega a más del $3 \%$ de la superficie de la tierra.

Según este discurso, las grandes ciudades, por su alta densidad, son más eficientes en el uso de los recursos. El economista Edward Glaeser sostiene que la ciudad de Nueva York es un modelo por su alta densidad, su sistema de transporte público y la presencia de nuevos rascacielos con sistemas de calefacción y aire acondicionado más eficientes (Glaeser, 2011). Glaeser, siendo admirador de la magia del mercado, el régimen neoliberal y también amigo del alcalde de Nueva York, el billonario Michael Bloomberg, está enfocado en la pequeña isla de Manhattan, que representa apenas dos millones de una población de veinte, en un área metropolitana que es una de las más dispersas en los EE.UU., aún más que la famosa Los Ángeles. Los admiradores de la ciudad vertical notan que los edificios nuevos son más eficientes en el "uso del suelo" (los expertos reflejan claramente el concepto colonial del "uso de la tierra") y en el uso de la energía, afirmando que los centros urbanos más densos permiten la construcción de sistemas de transporte urbano más eficientes. Pero también parecería que piensan que la solución de los problemas ambientales es más factible en los centros densos como Manhattan, por la misma concentración de capital.

Este mito de la alta densidad está fuertemente promovido por los bancos e inversionistas globales que están invirtiendo importantes excedentes de capital en los grandes centros urbanos. Estamos en la época de la financiarización excesiva de capital a nivel global donde el sector inmobiliario es efectivamente uno de los mayores ganadores.

Estos mitos se contraponen a la realidad de Nueva York y de otros centros urbanos del norte. Como la mayoría de las grandes ciudades del mundo, Nueva York fue fundada al lado de los océanos y grandes vías fluviales para facilitar la extracción de los minerales y productos industriales.

Hay un fuerte incentivo en solucionar el problema de los gases de efecto invernadero por ser las ciudades más vulnerables a las inundaciones causadas por el aumento del nivel del mar.

Pero Nueva York es una prueba de la falsedad del mito de la densidad. Examinando el sistema de transporte público, el más grande de América del Norte, se pone de manifiesto que este sistema cubre mucho menos de la mitad del territorio en el área metropolitana. La unidad política de la ciudad de Nueva York, donde viven más de ocho millones de personas, es solo la parte central del área metropolitana que cuenta con más de 20 millones de personas.

En la metrópoli la mayoría de los traslados se hacen con el automóvil. Después de la crisis financiera de 2008 el gobierno nacional salvó la industria automotriz y el país sigue manteniendo una forma de movilidad que hace que los ciudadanos mantengan la experiencia de conocer el territorio principalmente desde la perspectiva de una máquina nueva. 
"A principios de este siglo, las encuestas internacionales revelaron datos elocuentes: la mayoría de la gente contestó que la peor desgracia que te puede ocurrir es que te roben el automóvil y no puedas recuperarlo." Eduardo Galeano, El Cazador de Historias. 2016.

Los ciudadanos de Nueva York que usamos el sistema de transporte público somos conscientes que la infraestructura fue construida hace más de un siglo y que no se corresponde con las necesidades actuales. También New York posee un sistema de saneamiento tan inadecuado que cada vez que llueve la mayoría de los efluentes se descarga directamente a los ríos y las bahías. $Y$ hay muchos otros ejemplos del retraso de Nueva York y otros centros urbanos construidos para beneficio del capital en vez de las personas, reflejado en el uso sostenible de los recursos naturales, en la contaminación ambiental, y sobre todo en la calidad de vida (Angotti, 2008).

Pero en Nueva York y en todo el mundo hay una división siempre más fuerte entre las ciudades y las zonas protegidas y las áreas que sufren las peores consecuencias del cambio climático. Los centros densamente poblados del presente y futuro serán enclaves protegidos para aquellos que tienen más recursos económicos y en la periferia los demás tendremos que intentar salvarnos. Esta es la problemática de la justicia ambiental, muchos barrios habitados por minorías étnicas son los más vulnerables al desplazamiento y que no se benefician de las alternativas propuestas por las entidades públicas y privadas (Sze, 2006).

\section{EL PLAN DE LOS ENCLAVES}

En 2007 la ciudad de Nueva York, bajo el gobierno del alcalde Bloomberg, uno de los hombres más ricos del mundo, publicó un Plan Sostenible basado en muchos de los mitos de la alta densidad (Angotti, 2007). El plan recomendó la expansión del centro, en particular la isla de Manhattan y sus alrededores donde los rascacielos son notables, para permitir el alojamiento de más de un millón de nuevos habitantes. Se trataba de un plan para la concentración de la población en el área central y su justificación radicaba en lograr una ciudad "mejor" y "más verde". Pocos años después la ciudad sufrió dos grandes inundaciones, probablemente relacionadas con el cambio climático y a la visión futurista de Bloomberg se añadió la idea de proteger las propiedades de alto valor en el Bajo Manhattan mediante la construcción de barreras enormes, seguramente a precios astronómicos.

En esto consistía el plan de enclave, la solución preferida por el capitalismo global. Es la idea del Arca de Noé, una continuación de la práctica de construir malls y comunidades cerradas, la de crear reservas naturales. Y también en una forma menos dramática es el proceso de "gentrificación verde", lo que queda por fuera de estos nuevos enclaves protegidos serán las zonas de sacrificio.

Los enclaves protegidos están construidos mediante inversiones privadas, pero con incentivos de los sectores públicos. Muchos de ellos están en la costa, en áreas estratégicas 
para las inversiones comerciales, el transporte marítimo y el turismo. Tal vez uno de los iconos en los Estados Unidos sea Mar-a-Lago, el lujoso resort en la costa sur del estado de Florida, propiedad de Donald Trump.

Por supuesto el modelo de los enclaves no es nuevo. La historia urbana de las Américas es uno de enclaves coloniales protegidos donde se concentraron los recursos e inversiones de capital, en tanto la mayoría de la población indígena y los trabajadores humildes se quedaron fuera de las áreas centrales planificadas. El modelo de los enclaves domina la planificación moderna, con los malls y comunidades cerradas incluso en las áreas rurales, donde están las reservas naturales. También está el producto del "capitalismo de desastres" (Klein, 2014) cuando en New Orleans después del Huracán Katrina en 2005, miles de desplazados pobres y negros quedaron fuera del nuevo enclave urbano reconstruido (Hartman and Squires, 2006).

\section{LA PLANIFICACIÓN NEOLIBERAL}

El monopolio del sector privado en materia de planificación urbana y territorial fue consolidado con la planificación neoliberal. Al principio del siglo pasado, cuando nacieron las primeras metrópolis en el mundo, la planificación integral y racional, auspiciada por entidades del estado, dominaba en las profesiones urbanas. En la segunda mitad del siglo la planificación integral fue reemplazada por la planificación neoliberal. Ya no pensamos en las utopías urbanas, ni las discutimos, ni pensamos en el futuro lejano. En la actualidad, la planificación está reducida a un horizonte de 5 o 10 años, como máximo 20 a 30 años, para inversiones en el mercado financiero e inmobiliario.

En el lugar de los instrumentos integrales-racionales apareció en las últimas décadas del siglo pasado, el plan estratégico. La planificación estratégica se originó en los Estados Unidos dentro del mundo de la planificación militar y el mundo de business. Con la transición de la administración pública desde el welfare state hacia el neoliberalismo, la planificación estratégica fue adoptada como modelo preferido. Los planes maestros de la planificación tradicional fueron siempre muy detallados, complicados y accesibles solo para los técnicos, arquitectos, ingenieros y algunos funcionarios públicos. Pero a medida que la metrópolis crecía y se fue complicando la planificación, los planes estratégicos entraron en escena y redujeron las complejidades a unos conceptos y fórmulas muy fáciles. El nuevo plan estratégico muchas veces sirve como discurso mágico que no pone en la mesa los acuerdos ya hechos con las empresas que comandan el verdadero proceso de desarrollo urbano, los "stakeholders" (o mejor dicho los dueños de las propiedades urbanas).

Después de la primera conferencia mundial sobre el ambiente en Rio, en 1992, la planificación estratégica fue adoptada como la metodología preferida para guiar la política pública y privada en cuestiones del ambiente, la sostenibilidad, la resiliencia y el cambio climático. La Agenda 21, proceso de planificación local que debía desarrollar estrategias para los gobiernos locales, muchas veces llega a adoptar visiones al largo plazo, pero están 
subordinadas a las decisiones de corto plazo de los gobiernos locales. La llegada del concepto de sostenibilidad fue positiva porque proponía la cuestión de control sobre el futuro, siendo un desafio en sus comienzos, al principio neoliberal en cuanto a que "no hay alternativa" al capitalismo y el "libre mercado." Pero los planes de sostenibilidad muchas veces siguieron intereses económicos, muy pocos preguntaron quiénes serían los beneficiarios del plan y quiénes iban a perder.

Con los primeros desastres vinculados al cambio climático, inundaciones, sequías y huracanes, resulta obvio que haya muchas ciudades y áreas rurales vulnerables a los cambios y esto implica comprender los factores que producen lugares y poblaciones resilientes. Pero los planes de resiliencia también reducen a fórmulas y políticas de cambio parcial y de corto plazo, cuestiones sistemáticas muy complicadas (hay muchas teorías e interpretaciones de la resiliencia). Muchas de las poblaciones afectadas por los desastres rechazan la interpretación que la resiliencia significa volver al estado que existía antes cuando se trataba de un estado de extrema desigualdad social y económica.

\section{GESTIÓN DE BIENES COMUNES Y BUENAS PRÁCTICAS}

Otra novedad es la de buenas prácticas, innovaciones que nacen en forma aislada, como por ejemplo el presupuesto participativo de Porto Alegre en Brasil y el urbanismo social de Medellín, Colombia. Se han premiado y vendido en todo el mundo como mercancía, elementos esenciales en el concurso global entre ciudades competitivas, son ciudades a la venta en el mercado global.

Todas estas invenciones forman parte del mundo contemporáneo de las soluciones tecnológicas. Lejos de tener un enfoque ecosistémico, estas soluciones son parciales y muchas veces sirven para fraccionar la realidad urbana y reducir cuestiones de profundo interés social y económico, a nuevas formas de exclusión social.

En su importante encíclica, Laudato Si (2015), el Papa Francisco escribe:

“...un enfoque realmente ecológico debe transformarse en un enfoque social, que debe integrar la justicia en las conversaciones sobre medioambiente, para escuchar el pianto de la tierra y de los pobres".

\section{¿QUÉ HACER?}

En este ensayo mi énfasis ha estado en el análisis y la crítica y no he propuesto alternativas. Creo que hay muchísimas opciones tangibles y visibles que nacen de los movimientos y fuerzas de oposición en todo el mundo. Hay que escuchar las voces de los que no tienen micrófonos, los que siguen luchando no solo por sus derechos a la tierra sino también por los derechos a la tierra y la naturaleza.

Entonces podremos, 
Vivir con la tierra y no sobre ella

Promover la propiedad en común y con una variedad de expresiones (Ostrom, 1990)

Crear la gestión y cogestión de los bienes comunes

En vez de buenas prácticas, hay que intercambiar nuestras prácticas

Avanzar en Justicia climática

Luchar por el derecho a la ciudad y el derecho al campo

Reconocer los derechos de la naturaleza (Morales Ayma, 2011)

Planificar a largo plazo (por siete generaciones)

Planificación con diálogo y no solo con la participación (Freire, 2002)

Planificar no solo el espacio sino también el tiempo

En fin, en lugar de la ciudad neoliberal y la ciudad de enclaves, que se ocupan de la dimensión del espacio, hay que pensar y actuar a favor de la ciudad lenta, donde el tiempo y el espacio estén dirigidas hacia el universo más justo y más lento. Según el Papa Francisco en Laudato Si:

"Las diferencias entre tiempo y espacio, con la nueva tecnología se minimizan. Pero la velocidad máxima está causando una separación aún más grande entre los humanos y la tierra." 


\section{BIBLIOGRAFÍA}

Angotti, T. (2008). Is New York's Sustainability Plan Sustainable? Paper presented to the joint conference of the Association of Collegiate Schools of Planning and Association of European Schools of Planning (ACSP/ AESOP), Chicago.

Angotti, T. (2008). New York For Sale. Cambridge: MIT Press.

Angotti, T. (2012). The New Century of the Metropolis: Urban Enclaves and Orientalism. Routledge.

Angotti, T. (2013). Urban Latin America: Violence, Enclaves and Struggles for Land. En: Latin American

Perspectives. (40)2, 5-20.

Angotti, T. (ed.) (2017). Urban Latin America: Inequalities and Neoliberal Reforms. Rowman Littlefield.

Freire, P. (2002). Pedagogy of the Oppressed. New York: Continuum.

Glaeser, E. (2011). The Triumph of the City. New York: Penguin Press.

Harnecker, M. (2005). Haciendo Camino al Andar. Milenio Libre.

Hartman, Ch., Squires, G. D. (2006). There Is No Such Thing as a Natural Disaster. New York: Routledge.

Klein, N. (2014). This Changes Everything: Capitalism vs. the Climate. Simon \& Schuster. Morales Ayma, E. et al. (2011). The Rights of Nature. Global Exchange.

Ostrom, E. (1990). Governing the Commons. Cambridge University Press. 